\title{
DIVERSIDADE DA MIRMECOFAUNA EM UMA ÁREA DEGRADADA PELA GARIMPAGEM DE DIAMANTES NO ESTADO DE MATO GROSSO, BRASIL ${ }^{1}$
}

ROCHA, Wilian de Oliveira. Engenheiro florestal, discente do Programa de Pós-Graduação em Ciências Florestais e Ambientais da Universidade Federal de Mato Grosso e Docente do Centro Universitário de Várzea

Grande - UNIVAG. Av. Dom Orlando Chaves, 2655, Cristo Rei - Várzea Grande-MT. CEP 78118-900.

E-mail: wilianroch@ hotmail.com.

DORVAL, Alberto. Doutor, docente do Programa de Pós-Graduação em Ciências Florestais e Ambientais e Graduação em Engenharia Florestal da Universidade Federal de Mato Grosso. E-mail: adorval@terra.com.br. PERES FILHO, Otavio. Doutor, docente do Programa de Pós-Graduação em Ciências Florestais e Ambientais e Graduação em Engenharia Florestal da Universidade Federal de Mato Grosso.

E-mail: o.peresfilho@yahoo.com.br.

VAEZ, Caroline Dos Anjos. Discente em Engenharia Florestal pela Universidade Federal de Mato Grosso.

E-mail: carol_anjosvaez@hotmail.com.

CASTRO, Cibele Kotsubo da Cunha. Estagiária/Pesquisadora da O.N.F. Brasil e discente do Programa de PósGraduação em Ciências Florestais e Ambientais da Universidade Federal de Mato Grosso. Av. Fernando Corrêa da Costa, 2367, Bairro Boa Esperança, Cuiabá-MT, CEP 78060-900. E-mail: cibelekotsubo@ hotmail.com.

\section{RESUMo}

Este trabalho teve por objetivo amostrar a diversidade de formigas em ambientes influenciados pelas atividades de garimpagem de diamantes no Cerrado, disponibilizando informações sobre a mirmecofauna nesta região de Mato Grosso. O estudo foi realizado no município de Poxoréu-MT, em propriedade com garimpagem de diamantes, onde foram instaladas armadilhas "pitfall" em quatro transectos lineares distintos por ambiente: A1 ambiente degradado pela garimpagem de diamantes, A2 - ambiente adjacente a A1 apresentando vegetação de Cerrado com influência antrópica, A3 - ambiente adjacente a A2 com vegetação de Cerrado antropizada, fazendo limite com A4 - ambiente com vegetação de Cerrado preservada sem alterações antrópicas. As coletas foram realizadas bimestralmente em um período de um ano, sendo as amostras quantificadas e identificadas em nível de espécie para que fossem aplicados os índices de diversidade para cada ambiente. Foram amostradas 15 espécies de formigas dentre os quatro ambientes, sendo 9 espécies em A1, 13 em A2, as 15 em A3 e 12 espécies em A4, apresentando a espécie Brachymyrmex patagonicus como a mais abundante, forrageando em todos os ambientes. Os índices de diversidade permitem concluir que A3 possui a maior diversidade de espécies, e inversamente a menor dominância destas, entre todos os ambientes. Já A1 demonstrou a maior dominância das espécies de formigas em função da menor diversidade destas. Assim, a degradação ambiental pelo garimpo de diamantes pode afetar a composição da diversidade de formigas e sua dominância em meio à vegetação de Cerrado.

PalaVras-Chave: Formigas; Degradação ambiental; Armadilhas "pitfall".

\footnotetext{
${ }^{1}$ Artigo retirado de dissertação de Mestrado em Ciências Florestais e Ambientais - UFMT.
} 
ANT FAUNA DIVERSITY IN AN AREA DEGRADED BY diamond mining in the State of Mato Grosso, BRAZIL

\section{Abstract}

This study aimed at sampling the diversity of ants in environments influenced by diamond mining activities in the Brazilian savannah, providing information on the ant fauna in this region of Mato Grosso.The study was carried out in the city of Poxoréu-MT, Brazil, in a property with diamond mining, where pitfall traps were installed in four different transects per environment: A1 - degraded area by diamond mining $\mathrm{A} 2$ - adjacent area to A1 with savannah vegetation with anthropogenic influence, A3 - adjacent area to A2 with anthropized savannah vegetation, bordering with A4 - area with savannah vegetation preserved without anthropogenic changes. Samples were collected bimonthly for a period of one year, and the samples were quantified and identified taking into account their species level in order to allow the application of the diversity indices for each environment. 15 species of ants were sampled among the four environments, being nine species in $\mathrm{A} 1,13$ in A2, 15 in A3 and 12 species in A4, presenting Brachymyrmex patagonicus as the most abundant, foraging in all environments. Diversity indices allow to conclude that A3 has the greatest diversity of species, and conversely the lower dominance, in all environments. However, A1 was the most dominant species of ants due to the lowest diversity. Thus, environmental degradation by diamond mining can affect the composition of ants and their dominance in the savannah vegetation.

KEYwORDS: Ants, Environmental degradation, Pitfall traps.

\section{INTRODUÇÃO}

As explorações dos ecossistemas resultam na diminuição da densidade e diversidade de uma vasta quantidade de organismos que habitam o solo e que são fundamentais na decomposição de matéria orgânica e ciclagem de nutrientes (LIMA et al., 2006). Dessa forma, a qualidade do solo em declínio, e/ou sua total degradação, promovem a redução de biodiversidade animal, principalmente de invertebrados, afetando a manutenção dos ecossistemas terrestres como um todo (AZEVEDO, 2004).

As formigas se constituem em um dos grandes grupos de invertebrados nas regiões tropicais, pois apresentam alta riqueza de espécies e são ecologicamente importantes na serapilheira de espécies florestais tropicais (AGOSTI et al. 2000).

A riqueza e a diversidade de espécies de formigas podem ser maiores em ambientes de complexidade mais elevada, devido a uma maior disponibilidade de nichos presentes. Em razão da conexão entre os organismos e as características dos habitats, as formigas têm sido utilizadas como ferramentas no monitoramento ambiental de áreas perturbadas (PEREIRA et al., 2007), por apresentarem uma grande quantidade de característica desejáveis, como facilidade de amostragem, alta abundância e ampla distribuição, importância no funcionamento dos ecossistemas, ecologia e taxonomia relativamente bem conhecida (AGOSTI et al., 2000).

No Cerrado brasileiro, a riqueza e a densidade de árvores tendem a aumentar a variedade e a quantidade de recursos nos ambientes, possibilitando maior riqueza e maior número de espécies generalistas, podendo também provocar mudanças no habitat e na dominância da comunidade de formigas (RIBAS et al., 2003).

Diante de estudos e pesquisas, percebe-se que o inventário da fauna e da flora em geral, principalmente com a utilização de organismos como as formigas, em ambientes modificados sujeitos à degradação ou à reabilitação, é de grande importância ecológica para que decisões ambientalmente corretas possam ser tomadas.

Assim, este estudo objetivou amostrar a diversidade de formigas em ambientes influenciados pelas atividades de garimpagem de diamantes no Cerrado, disponibilizando informações sobre a mirmecofauna nesse tipo de degradação. 


\section{MATERIal e MÉTODOS}

O estudo foi realizado na Fazenda Limeira, município de Poxoréu, Mato Grosso, onde existem diversas áreas degradadas por atividade de garimpos de diamantes no bioma Cerrado.

A área amostral compreende a fitofisionomia Cerrado stricto sensu (ou sentido restrito), caracterizada pela presença de árvores baixas, inclinadas e tortuosas, onde estão presentes arbustos e subarbustos espalhados em meio ao estrato herbáceo, composto predominantemente por gramíneas (RIBEIRO E WALTER, 1998).

O clima é tropical quente e sub-úmido (Aw, segundo Köppen), com temperatura média anual de $22^{\circ} \mathrm{C}$ e precipitação anual de $1750 \mathrm{~mm}$ a $1900 \mathrm{~mm}$, tendo intensidade máxima nos meses de dezembro, janeiro e fevereiro. Aaltitude média é de $450 \mathrm{~m}$ acima do nível do mar. O solo da região varia entre Latossolo vermelho amarelo e Latossolo vermelho escuro, com média a baixa fertilidade. (PREFEITURA MUNICIPAL DE POXORÉU, 2009).

Nessa área predominam quatro ambientes distintos, apresentando um gradiente de degradação ambiental promovido pelas atividades do garimpo de diamantes, sendo: Ambiente 1 (A1) - Área degradada pela garimpagem de diamantes, com vegetação totalmente removida e solos expostos, apresentando alto nível de erosão; Ambiente 2 (A2) - Área adjacente ao "Ambiente 1", com vegetação ainda estabelecida, porém foi antropizada pela instalação e vivência dos trabalhadores do garimpo ("garimpeiros") cerca de 10 anos atrás. Encontra-se limitada pela estrada que secciona ("corta") a área de estudo; Ambiente 3 (A3) - Área adjacente ao "Ambiente 2", sendo separado deste, por um lado, pela estrada que secciona a propriedade e delimitada, de outro lado, pela área de Cerrado inteiramente preservado. Apresenta, assim como o ambiente anterior, vegetação ainda estabelecida com vestígios da presença humana dos garimpos provenientes de 30 anos atrás; Ambiente 4 (A4) - Área adjacente ao "Ambiente 3", com vegetação preservada, solo inteiramente coberto por serapilheira e pelo dossel típico do Cerrado sensu stricto, e visível ausência de antropização.

Em cada ambiente foi instalado um transecto linear de 150 metros de comprimento, onde foram distribuídas 11 armadilhas do modelo "pitfall", equidistantes 15 metros entre si.

As armadilhas "pitfall" foram confeccionadas com potes plásticos transparentes de $300 \mathrm{ml}$, com $15 \mathrm{~cm}$ de altura e $10 \mathrm{~cm}$ de diâmetro. Foram enterradas com suas bordas ao nível da superfície do solo, sendo preenchidas com solução conservante contendo água, detergente neutro e sal, a um terço da capacidade do recipiente (AQUINO et al., 2006). Após o período de 24 horas, as armadilhas foram retiradas e o conteúdo coletado foi devidamente individualizado por armadilha, armazenado e etiquetado com as características do local. Foram realizadas coletas bimestrais, no período de junho de 2010 a abril de 2011, totalizando 6 coletas e 264 amostras.

Os espécimes coletados foram transportados ao Laboratório de Proteção Florestal (LAPROFLOR), da Faculdade de Engenharia Florestal (FENF), da Universidade Federal de Mato Grosso (UFMT), para triagem e contagem das formigas. Os espécimes morfologicamente diferentes foram enviados ao dr. Jacques Hubert C. Delabie - Laboratório de Mirmecologia do Centro de Pesquisas do Cacau (CEPEC) da Comissão Executiva do Plano da Lavoura Cacaueira (CEPLAC), Ilhéus, Bahia - para a devida identificação taxonômica em nível específico.

Para a análise dos dados coletados foram aplicados os seguintes índices de diversidade:

- Índice de Riqueza:

Foi calculado o Índice de Riqueza de Margaleff, que demonstra a riqueza de espécies existentes dentro de cada área e expressa a diversidade de espécies, ou seja, refere-se à abundância de uma determinada área geográfica, região ou comunidade (RODRIGUES et al., 2004).

$$
\text { R1= (S - 1)0,4343 / }\left(\log _{10} N\right) \text {, em que: }
$$

$\mathrm{S}=\mathrm{N} .{ }^{\circ}$ de espécies presentes no ambiente; 
$\log _{10} \mathrm{~N}=$ logaritmo (base 10) do número de indivíduos presentes no ambiente.

- Índice de diversidade:

Para o estudo deste parâmetro, foi utilizado o índice de Ludwig e Reynolds (1988), que traz os números de diversidade de Hill (N0, N1 e N2), os mais recomendados para medir a diversidade de espécies, por serem mais interpretativos que outros índices de diversidade e terem a vantagem de sua unidade de medida ser dada em espécie.

Equação de Hill:

Número 0: N0 = S

Número 1: $\mathbf{N 1}=\mathrm{e}^{\mathrm{H}^{\prime}}$

Número 2: $\mathbf{N 2}=1 / \mathrm{h}$, em que:

$\mathrm{S}=$ número total de espécies consideradas;

$\mathrm{H}^{\prime}=$ índice de Shannon, que transformado para N1, refere-se ao número de espécies abundantes.

$\mathbf{H}^{\prime}=-\Sigma($ pi ln pi), em que:

pi = proporção de indivíduos da i-ésima espécie.

$\mathrm{h}=$ índice de Simpson, que transformado para N2, refere-se ao número de espécies muito abundantes:

$$
\mathbf{h}=\Sigma \mathbf{n i}(\mathbf{n i}-\mathbf{1}) / \mathbf{n}(\mathbf{n}-\mathbf{1}), \text { em que: }
$$

$\mathrm{S}=$ número total de espécies consideradas;

$\mathrm{n}=$ número total de indivíduos da população; e

ni = número de indivíduos em cada uma das i-ésimas espécies.

- Índice de Equitatividade:

O índice de equitatividade foi obtido, por meio da relação entre N2 e N1, conhecida como Razão de Hill Modificada, conforme a fórmula:

$$
\mathbf{E}=(\mathbf{N} 2-\mathbf{1}) /(\mathbf{N 1}-\mathbf{1}), \text { em que: }
$$

$\mathrm{E}=$ equitatividade;

$\mathrm{N} 1$ = número 1 da diversidade de Hill; $\mathrm{e}$

N2 = número 2 da diversidade de Hill.

- Análise de Agrupamento ou "Cluster":

O estudo de similaridade entre ambientes foi feito através da análise de agrupamento ou "cluster", utilizando o Software Statistic 7.0, usando o diagrama da árvore, através do método de "Unweighted pairgroup average" e a distância euclidiana.

\section{Resultados e Discussão}

Nos quatro ambientes amostrados ocorreram as subfamílias Dolichoderinae, Ectatomminae, Formicinae, Myrmicinae e Ponerinae, sendo Formicinae a mais representativa em quantidade de espécies e de indivíduos coletados, independente do ambiente amostrado. Nesses quatro ambientes, foram coletados 10.521 indivíduos, sendo $2.665(25,30 \%)$ espécimes em A1, 3.625 (34,42\%) em A2, 1.934(18,36\%) em A3 e 2.307 (21,90\%) em A4 (Tabela 1).

O estudo obteve 15 espécies coletadas considerando todos os ambientes, e A3 foi o único que apresentou todas essas espécies. E o ambiente degradado pela garimpagem apresentou a menor quantidade de espécies (Tabela 1).

A subfamília Formicinae, como a mais diversificada em quantidade de espécies, apresentou Brachymyrmex patagonicus, Brachymyrmex pilipes, Camponotus (Myrmaphaenus) sp. 1, Camponotus crassus e Camponotus vittatus nos quatro ambientes estudados, enquanto, Camponotus melanoticus ocorreu nos ambientes A2, A3 e A4, e Camponotus atriceps somente em A3 e A4 (Tabela 1).

No entanto, dentre as quatro subfamílias, Dolichoderinae foi a menos diversificada em espécies, já que apresentou somente Forelius brasiliensis, que não foi encontrada no Ambiente 4, estando presente nos demais ambientes (Tabela 1). 
Tabela 1 - Sub-família, espécies e quantidade de indivíduos coletados com armadilhas pitfall nos quatro ambientes, Poxoréu, MT. Junho de 2010 a abril de 2011.

\begin{tabular}{|c|c|c|c|c|c|c|}
\hline \multirow{2}{*}{ Subfamílias/ Espécies } & A1 & A2 & A3 & A4 & \multirow[b]{2}{*}{ Total } & \multirow[b]{2}{*}{$\%$} \\
\hline & I & I & $\mathrm{I}$ & I & & \\
\hline \multicolumn{7}{|l|}{ Dolichoderinae } \\
\hline Forelius brasiliensis & 152 & 14 & 123 & - & 289 & 2,75 \\
\hline \multicolumn{7}{|l|}{ Ectatomminae } \\
\hline Ectatomma edentatum & - & 2 & 8 & - & 10 & 0,10 \\
\hline Ectatomma opaciventre & 53 & 49 & 40 & 14 & 156 & 1,48 \\
\hline \multicolumn{7}{|l|}{ Formicinae } \\
\hline Brachymyrmex patagonicus & 1.771 & 1.759 & 483 & 1.402 & 5.415 & 51,47 \\
\hline Brachymyrmex pilipes & 145 & 1.017 & 8 & 70 & 1.240 & 11,79 \\
\hline \multicolumn{7}{|l|}{ Camponotus } \\
\hline (Myrmaphaenus) sp. & 374 & 135 & 163 & 42 & 714 & 6,79 \\
\hline Camponotus atriceps & - & - & 74 & 289 & 363 & 3,45 \\
\hline Camponotus crassus & 1 & 47 & 325 & 150 & 523 & 4,97 \\
\hline Camponotus melanoticus & - & 246 & 397 & 121 & 764 & 7,26 \\
\hline Camponotus vittatus & 20 & 14 & 2 & 6 & 42 & 0,40 \\
\hline \multicolumn{7}{|l|}{ Myrmicinae } \\
\hline Cephalotes pusillus & 10 & - & 3 & 1 & 14 & 0,13 \\
\hline Solenopsis substituta & 129 & 271 & 216 & 70 & 686 & 6,52 \\
\hline Trachymyrmex sp.1 & - & 9 & 46 & - & 55 & 0,52 \\
\hline \multicolumn{7}{|l|}{ Ponerinae } \\
\hline Pachycondyla crassinoda & - & 52 & 22 & 86 & 160 & 1,52 \\
\hline Paraponera clavata & - & 10 & 24 & 56 & 90 & 0,86 \\
\hline TOTAL & 2.655 & 3.625 & 1.934 & 2.307 & 10.521 & 100 \\
\hline
\end{tabular}

A1: Ambiente 1; A2: Ambiente 2; A3: Ambiente 3; A4: Ambiente 4; I: Indivíduos; \%: Porcentagem de indivíduos.

Fonte: Dados de pesquisa. 
Observou-se a ocorrência de sete espécies comuns a todos os ambientes estudados, em que cinco são Formicinae, demonstrando a capacidade dessa subfamília em se adaptar a ambientes distintos. Uma espécie comum a todos os ambientes, mas que pertence à subfamília Myrmicinae, é Solenopsis, substituta que apresentou uma quantidade total de indivíduos coletados consideravelmente expressiva diante das demais espécies (Tabela 1).

A maior incidência de espécies da subfamília Formicinae, nidificando em quase todos os ambientes da área de estudo (Tabela 1), pode estar relacionada ao fato de que, muitas vezes, essa subfamília é afetada pela presença ou ausência de espécies do gênero Pheidole em áreas tropicais, possivelmente por competirem pelos mesmos recursos (FOWLER, 1993; OLIVEIRA, 2009).

O fato foi demonstrado por Rocha (2010), em pesquisa na região, quando observou alta dominância, abundância e frequência de Pheidole spp., enquanto as duas espécies de Camponotus spp. ocorreram como não dominantes, comuns e nível médio de frequência. Assim, a ausência do gênero Pheidole nessa área (Tabela 1) permitiu inferir que o fato contribuiu para a maior riqueza específica de Formicinae, quando comparada com as demais subfamílias.

As espécies do gênero Camponotus, compondo a maior parte de Formicíneas coletadas, são onívoras, geralmente nidificando em galhos de árvores e são consideradas pouco agressivas, sendo encontradas de forma ampla no bioma de Cerrado e em ambientes urbanos (FOWLER, 1991; BOLTON, 2003; ROCHA e SOLFERINI, 2008), o que pode explicar a ocorrência desse gênero em todos os ambientes amostrados, desde os antropizados até o Cerrado preservado.

As espécies do gênero Brachymyrmex são consideradas forrageiras dominantes de serapilheira (RÉ, 2007), de hábito alimentar onívoras (DELABIE et al., 2000), o que possibilitou o estabelecimento de colônias em todos os ambientes amostrados.

A subfamília Myrmicinae, apesar de ser considerada a mais abundante na região Neotropical, devido a sua capacidade de se adaptar a diferentes condições ambientais e disponibilidade de recursos (FOWLER, 1993), apresentou somente três espécies, sendo a $S$. substituta a mais expressiva em quantidade de indivíduos coletados, e a única dentre essas a se distribuir em todos os ambientes.

A espécie $S$. substituta, conhecida popularmente como formiga-lava-pés, demonstra grande adaptabilidade ambiental, pois ocorreu em todos os ambientes estudados, sendo consideradas formigas de hábitos generalistas (RÉ, 2007).

Várias espécies da subfamília Dolichoderinae são onívoras, forrageando sobre a superfície do solo, onde a alimentação consiste de insetos mortos, cera e exsudados de plantas (JAIME, 2010). Assim, a única espécie coletada dessa subfamília, F. brasiliensis, tem seu gênero considerado como especialista em clima quente (RÉ, 2007), com indivíduos muito ativos em áreas abertas, construindo seus ninhos próximos ou na base de arbustos (OLIVEIRA, 2009).

No entanto, surpreende a presença de $F$. brasiliensis, pois em estudos de Cuezzo (2000), esta espécie ocorre por toda a região sul da América do Sul estendendo-se até o Paraguai, mas não sendo relatada em Mato Grosso. Assim, a presença dessa espécie neste estudo pode apontar a ampliação de sua distribuição geográfica, sendo necessária a aplicação de outras pesquisas, no intuito de levantar dados sobre a ecologia da espécie na região.

Diante das amostragens coletadas, a partir das espécies de formigas, foram obtidos os índices de diversidade para comparar e diferenciar os quatro ambientes estudados.

No ambiente A1 ocorreram nove espécies, sendo três abundantes - F. brasiliensis, B. pilipes e $S$. substituta - e duas muito abundantes $-B$. patagonicus e Camponotus (Myrmaphaenus) sp. Os valores dos índices de Simpson e de Shanon mostram uma alta dominância das espécies presentes, devido à menor diversidade de espécies entre todos os ambientes amostrados. $\mathrm{O}$ valor da equitatividade expressa uma distribuição mediana dos indivíduos dessas espécies dentro do ambiente, que pode ser 
justificado pelo fato de a área degradada apresentar árvores pioneiras e arbustos isolados entre si, aglomerando em determinados pontos os indivíduos de cada espécie. $\mathrm{O}$ valor do índice de riqueza de Margalef mostra que esse ambiente se encontra muito alterado, com a estrutura da vegetação totalmente comprometida e o solo exposto, o que dificulta o estabelecimento de espécies mais exigentes por nidificação e forrageamento, favorecendo somente as formigas mais generalistas (Tabela 2).

No ambiente A2 ocorreram 13 espécies, com quatro abundantes e três muito abundantes $-B$. patagonicus e B. pilipes. Os índices de Simpson e de Shannon demonstram uma dominância específica um pouco menor, devido ao aumento na diversidade de espécies. A equitatividade indica uma distribuição homogênea dos indivíduos em cada espécie, indicando uma baixa competição pelos recursos ambientais (Tabela 2).

Amostrando o maior número de espécies dentre todos os ambientes estudados, no ambiente A3 ocorreram oito espécies abundantes e seis muito abundantes, destacando-se Camponotus melanoticus, além de B. patagonicus. Os valores dos índices de Shannon e de Simpson mostraram uma elevada diversidade, baixa dominância entre as espécies, devido à distribuição homogênea dos recursos ambientais entre as populações de formigas nesse ambiente. Diante da maior equitatividade dentre todos os ambientes amostrados, o ambiente A3 apresenta melhor distribuição dos indivíduos de cada espécie, com um ambiente mais diversificado na oferta de recursos alimentares, onde há a interação de microhabitats, alguns provenientes da antiga antropização do garimpo e outros mais preservados, influenciados diretamente pela orla do Cerrado preservado (A4). Assim, há maior diversidade, além de maior riqueza de espécies, expressada pelo índice de Margalef, pois esse ambiente permite a interação tanto das espécies de formigas adaptadas à área degradada quanto à área de vegetação preservada (Tabela 2).

Tabela 2 - Índice de diversidade, de acordo com a série de números de Hill, equitatividade e de Margalef, das espécies de formigas coletadas na Fazenda Limeira, município de Poxoréu - Mato Grosso - Brasil. Junho de 2010 a abril de 2011.

\begin{tabular}{l|c|c|c|c|c|c|c}
\hline \multirow{2}{*}{ AMBIENTES } & \multicolumn{7}{|c}{ INDICE DE DIVERSIDADE } \\
\cline { 2 - 9 } & $\mathrm{N}_{0}$ & $\mathrm{~N}_{1}$ & $\mathrm{~N}_{2}$ & $\chi$ & $\mathrm{H}^{\prime}$ & $\mathrm{E}$ & $\mathrm{R} 1$ \\
\hline A1 & 9 & 3,172 & 2,111 & 0,473 & 1,154 & 0,511 & 1,044 \\
A2 & 13 & 4,306 & 3,065 & 0,326 & 1,460 & 0,624 & 1,464 \\
A3 & 15 & 7,859 & 6,300 & 0,158 & 2,061 & 0,801 & 1,850 \\
A4 & 12 & 4,233 & 2,525 & 0,395 & 1,442 & 0,471 & 1,420 \\
\hline
\end{tabular}

$\mathbf{N}_{0}$ : número total de espécies; $\mathbf{N}_{1}$ número de espécies abundantes; $\mathbf{N}_{2}$ : número de espécies muito abundantes; $\chi \chi$ : Índice de Simpson; H': Índice de Shannon; E: eqüitatividade entre as espécies; Rl: Índice de Margalef.

Ambientes: Al - Área degradada pelo garimpo; A2 - Bordadura da área degradada pelo garimpo com vegetação de cerrado (antropização recente); $\mathbf{A 3}$ - Bordadura da área de cerrado preservado (antropização antiga); A4 - Área de cerrado preservado (ausência de antropização).

Fonte: Dados de pesquisa. 
Observa-se, no ambiente A4, a ocorrência de 12 espécies, sendo 4 espécies abundantes e 3 muito abundantes, evidenciando Camponotus atriceps e Camponotus crassus, além de B. patagonicus. De acordo com os índices de Simpson e de Shannon, houve uma dominância média dos indivíduos de cada espécie, e isso se refletiu em menor equitatividade dentre todos os ambientes (Tabela 2). Essa menor equitatividade pode ser devido à distribuição populacional heterogênea das espécies nesse ambiente, ocasionada pela maior ocorrência daquelas mais específicas em relação às necessidades de recursos ecológicos, ou pela estrutura da vegetação de Cerrado estar passando do ponto de estabilidade para a senescência, cujo fato diminui recursos e aumenta competições intra e interespecíficas (OLIVEIRA, 2009).

Ao aplicar os resultados obtidos em uma análise de "Cluster", os Ambientes A1 e A4, estruturalmente diferentes em relação à vegetação e acúmulo de serapilheira, são demonstrados como similares ao considerar a quantidade de indivíduos e quantidade de espécies coletados (Figura 1). Este caso pode ser explicado devido à capacidade de resiliência do ambiente A1 de se regenerar a partir da degradação e abrigar populações de espécies mais generalistas, propiciando recursos para que suas colônias possam se estabelecer e colonizar o ambiente, contribuindo no processo de regeneração. Contudo, o ambiente A4 parece possuir uma estrutura da vegetação em níveis ecológicos estabilizados, em que a competição entre as espécies especialistas controla o crescimento exagerado das colônias e limita a dominância de novas espécies.

No entanto, essa similaridade ocorre devido à análise de agrupamento considerar somente o aspecto quantitativo desses ambientes, e não o aspecto qualitativo.

Foi observado que os ambientes A2 e A3, principalmente, estão distantes dos ambientes A1 e A4, ou seja, não possuem similaridades próximas (Figura 1). De acordo com Hölldobler e Wilson (1990), uma maior diversidade e riqueza de espécies de formigas são associadas com níveis intermediários de distúrbios, ou estágios de sucessão intermediária de áreas recuperadas ou regeneradas, onde a heterogeneidade da área é maior, sendo importante realizar levantamentos relativamente longos para definir de forma minuciosa as variações existentes. Essas afirmações são comprovadas neste estudo, onde as áreas intermediárias (A2 e A3), entre o ambiente degradado pela mineração de diamantes e o ambiente de vegetação não antropizada, apresentaram maior riqueza e maior diversidade de espécies, distanciandose desses dois ambientes extremos, que, segundo o agrupamento, são similares em nível de quantidade de espécies e composição das comunidades de formigas.

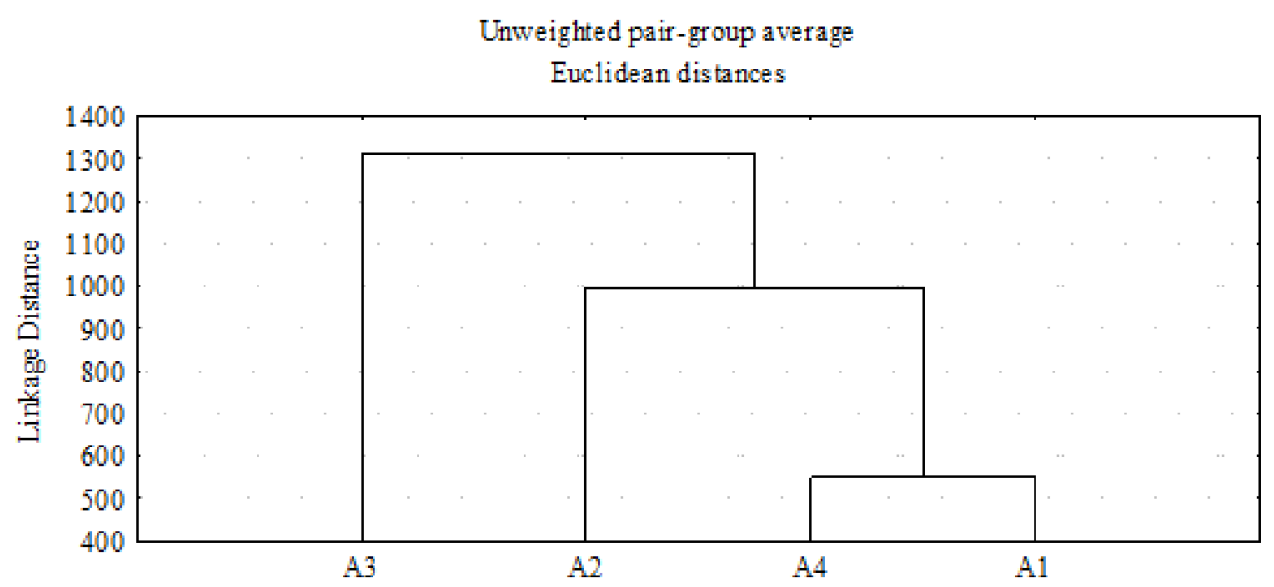

Figura 1 - Dendrograma entre os ambientes: (A1) área degradada; (A2) bordadura da área degradada; (A3) bordadura da área de vegetação de Cerrado; (A4) área com vegetação de Cerrado preservado; amostrados com armadilhas pitfall, na Fazenda Limeira, Poxoréu - Mato Grosso - Brasil. Junho de 2010 a abril de 2011.

Fonte: Dados de pesquisa. 


\section{Conclusão}

O Ambiente A3 apresentou maior diversidade de espécies entre todos os ambientes com a menor dominância destas na vegetação, e A1 demonstrou o contrário, maior dominância das espécies devido à menor diversidade destas. Assim, pode-se concluir que a degradação ambiental promovida pela atividade garimpeira interfere na diversidade de formigas no Cerrado que, consequentemente, modifica a dominância destas ao forragear esse tipo de vegetação.

\section{Agradecimentos}

Ao dr. Jacques Hubert C. Delabie e equipe do Laboratório de Mirmecologia do Centro de Pesquisas do Cacau (CEPEC) da Comissão Executiva do Plano da Lavoura Cacaueira (CEPLAC), Ilhéus, Bahia, pela identificação das espécies coletadas. Ao sr. Manoel Lauro da Silva, técnico do Laboratório de Proteção Florestal (LAPROFLOR) da Faculdade de Engenharia Florestal (FENF) da Universidade Federal de Mato Grosso (UFMT), pela acuidade com o material triado e conselhos para as coletas de campo. Ao Sr. Leônidas dos Santos por dispor sua propriedade para esta pesquisa.

\section{REFERÊNCIAS}

AGOSTI, D. et al. (Eds.). Ants: Standard methods for measuring and monitoring biodiversity.

Washington, D. C.: Smithsonian Institution Press, 2000. Disponível em: <http://antbase.org/databases/ publications_files/publication-20330.htm>. Acesso em: 01 ago. 2010.

AQUINO, A. M.; AGUIAR-MENEZES, E. L.; QUEIROZ, J. M. Recomendação para coleta de artrópodes terrestres por armadilhas de queda (pitfall traps). Seropédica, Rio de Janeiro: Embrapa Agrobiologia, 2006 (Circular Técnica, 18).

AZEVEDO, A.C. Funções ambientais do solo. In: AZEVEDO, A.C.de.; DALMOLIN, R.S.D.; PEDRON, F.de A. (Org.). Fórum Solos e ambiente, 1., 2004, Santa Maria: Pallotti, 2004. p.7-22.

BOLTON, B. Synopsis and classification of Formicidae. Memoirs of the American

Entomological Institute, Gainesville, v.71, p.1370. 2003.

CUEZZO, F. Revision del género Forelius (Hymenoptera: Formicidae: Dolichoderinae).

Sociobiology, California, v.35, n.2A, p. 197-275, 2000.

DELABIE, J.H.C.; AGOSTI, D.; NASCIMENTO, I.C. Litter and communities of the Brazilian Atlantic rain forest region. In: Agosti, D.; Majer, J.D.; Alonso, L.T. e Schultz, T. (ed.). Measuring and monitoring biological diversity: standart methods for ground living ants. Washington: Smithsonian Institution, 2000. p.280.

FOWLER, H. G. Ecologia nutricional de formigas. In: PANIZZI, A. R.; PARRA, J. R. P. (Eds).

Ecologia nutricional de insetos e suas implicações no manejo de pragas. São Paulo: Manole, 1991. p. 131-223.

FOWLER, H. G. Relative representation of Pheidole (Hymenoptera: Formicidae) in local ground ant assemblages of the Américas. Anales de Biologia, Murcia, v.19,n.8, p. 29-37, 1993.

HÖLLDOBLER, B.; WILSON, E. O. The ants. Harvard: University Press, 1990. 732p.

JAIME, N. G. Levantamentos mirmecofaunísticos em três ambientes antrópicos nos Estados de Goiás e Tocantins, Brasil. 2010. 131f. Tese (Doutorado). Universidade Federal de Goiás, Goiânia, 2010.

LIMA, A. A. de; LIMA, W. L. de; BERBARA, R. L. L. Diversidade da mesofauna de solo em sistemas 
de produção agroecológica. Revista Brasileira de Agroecologia, Porto Alegre, v. 1, n. 1, 2006. Porto Alegre. Disponívelem: $<\mathrm{http} / /$ www6.ufrgs.br/ seeragroecologia/ojs/viewarticle.php?id=290>. Acesso em: 01 set. 2009.

\section{LUDWIG, J. A.; REYNOLDS, J. F. Statistical} ecology: a primer on methods and computing. New York: John Wiley \& Sons, 1988. 337p.

OLIVEIRA, M. A. de. Diversidade da mirmecofauna e sucessão florestal na Amazônia -Acre, Brasil. 2009. 132 f. Tese (Doutorado) Universidade Federal de Viçosa, Viçosa, 2009.

PEREIRA, M. P. S. et al. Fauna de formigas como ferramenta para monitoramento de área de mineração reabilitada na Ilha da Madeira, Itaguaí, RJ. Ciência Florestal, Santa Maria, v. 17, n. 3, julset. 2007. Disponível em: < http:// redalyc.uaemex.mx/redalyc/pdf/534/ 53417302.pdf>. Acesso em: 01 set. 2009.

\section{PREFEITURA MUNICIPALDE POXORÉU -} MT. Nossa cidade: caracterização do município. Disponível em: <http://www.poxoreu.mt.gov.br/ nossacidade/caracterizacao.htm>. Acesso em: 14 fev. 2010.

\section{RÉ, T. M. O uso de formigas como}

bioindicadores no monitoramento ambiental de revegetação de áreas mineradas. 2007. 244 f. Tese (Doutorado) - Escola Politécnica da
Universidade de São Paulo, São Paulo, 2007.

RIBAS, C. R.; SCHOEREDER, J.H.; PIC, M.; SOARES, S.M. Tree heterogeneity, resource availability, and larger scale processes regulating arboreal ant species richness. Austral Ecology, Australia, v. 28, p.305-14. 2003.

RIBEIRO, J. F.; WALTER, B. M. T. Fitofisionomias do bioma Cerrado. In: SANO, S. M.; ALMEIDA, S. P. (Eds.). Cerrado: ambiente e flora. Planaltina: Embrapa Cerrados, 1998. p. 89-168.

ROCHA, R. A.; SOLFERINI, V. N. Número de rainhas e acasalamentos em Camponotus crassus (Mayr, 1862). CONGRESSO INTERNO DE INICIAÇÃO CIENTÍFICA, 16, 2008,

Campinas. Anais... Campinas, s. ed., set. 2008. Disponível em: <http://www.prp.unicamp.br/pibic/ congressos/xvicongresso/ cdrom/ FSCOMMAND/pdfN/341.pdf >. Acesso em: 20 jan. 2010.

ROCHA, W. O. Análise faunística da mirmecofauna em áreas degradadas pelo garimpo de diamantes no município de Poxoréu -MT. 2010. 38f. Monografia (Graduação) Universidade Federal de Mato Grosso, 2010.

RODRIGUES, S. T. et al. Composição florística e abundância de pteridófitas em três ambientes da bacia do rio Guamá, Belém,Pará, Brasil. Acta Amazonica, Manaus, v. 34,n.1, p. 35 - 42, 2004.

RECEBIDO EM 4/7/2015

ACEITO EM 9/12/2014 